\title{
ON THE CONVERGENCE OF SEQUENCES OF STOCHASTIC PROCESSES $\left({ }^{1}\right)$
}

\author{
BY \\ ERNEST G. KIMME
}

0 . Introduction. The purpose of this paper is the formulation and investigation of some convergence concepts for sequences of stochastic processes $\left\{x_{n}(t, \omega), t \in[0,1]\right\}$. A related result of these investigations appears as a generalization of the central-limit problem for sequences of sums of independent random variables, embodied in the discussions in $\S \S 3$ and 5 of the sequence (A); Gnedenko's necessary and sufficient conditions for the convergence in distribution of such sums are used and extended. $\$ 4$ contains a version of the Helly-Bray theorem (Theorem 9, Corollary) on probability spaces.

The tools used are those developed in $[2 ; 7 ; 8]$, and [9] for some special cases of convergence of stable processes. The methods of $\$ 4$ are a straightforward adaptation of those of [2]; the convergence property of $F[$ ] used in Theorem 9 was found to be necessary by Udagawa (in the publications of the Union of Japanese Scientists and Engineers) in considering the case of sequences $(\hat{A})$ of normed-sum type converging to non-Gaussian stable processes.

The processes are throughout this paper assumed to have independent increments. Some special cases of this convergence problem are considered in $[1 ; 3$; and 4$]$ without this condition; no general results seem to be known at present.

1. Notation and definitions. Let $(\Omega, B, p)$ denote a probability space. A real stochastic process defined on $(\Omega, \beta, p)$ with real parameter set $T$ we shall denote by $\{x(t, \omega), t \in T\}$. We shall consider sequences of stochastic processes $\left\{x_{n}(t, \omega), t \in T\right\}$, converging in some sense to a stochastic process $\{x(t, \omega)$, $t \in T\}$. Let

$$
\begin{aligned}
F\left(t_{1}, \cdots, t_{m} ; \lambda_{1}, \cdots, \lambda_{m}\right) & =p\left\{x\left(t_{j}, \omega\right) \leqq \lambda_{j}, 1 \leqq j \leqq m\right\} \\
F_{n}\left(t_{1}, \cdots, t_{m} ; \lambda_{1}, \cdots, \lambda_{m}\right) & =p\left\{x_{n}\left(t_{j}, \omega\right) \leqq \lambda_{j}, 1 \leqq j \leqq m\right\} \\
\phi\left(t_{1}, \cdots, t_{m} ; \mu_{1}, \cdots, \mu_{m}\right) & =E\left\{\exp \left(i \sum_{j=1}^{m} \mu_{j} x(t, \omega)\right)\right\} \\
\phi_{n}\left(t_{1}, \cdots, t_{m} ; \mu_{1}, \cdots, \mu_{m}\right) & =E\left\{\exp \left(i \sum_{j=1}^{m} \mu_{j} x_{n}\left(t_{j}, \omega\right)\right)\right\}
\end{aligned}
$$

where $t_{1}, \cdots, t_{m} \in T$.

Presented to the Society, April 28, 1956; received by the editors March 10, 1956.

(1) This paper contains the essential results of the author's doctoral thesis written under Professor M. D. Donsker at the University of Minnesota. 
We shall require two notions of convergence of processes:

Definition 1. The sequence $\left\{x_{n}(t, \omega), t \in T\right\}$ of stochastic processes converges in distribution to the stochastic process $\{x(t, \omega), t \in T\}$ if and only if for every positive integer $m$ and every sequence $\left(t_{1}, \cdots, t_{m}\right) \in T^{m}$

$$
\lim _{n \rightarrow \infty} F_{n}\left(t_{1}, \cdots, t_{m} ; \lambda_{1}, \cdots, \lambda_{m}\right)=F\left(t_{1}, \cdots, t_{m} ; \lambda_{1}, \cdots, \lambda_{m}\right)
$$

for every vector $\left(\lambda_{1}, \cdots, \lambda_{m}\right)$ of continuity of the limit function.

Definition 2. The sequence $\left\{x_{n}(t, \omega), t \in T\right\}$ converges uniformly in distribution to $\{x(t, \omega), t \in T\}$ if and only if for every positive integer $m$,

$$
\lim _{n \rightarrow \infty} \phi_{n}\left(t_{1}, \cdots, t_{m} ; \mu_{1}, \cdots, \mu_{m}\right)=\phi\left(t_{1}, \cdots, t_{m} ; \mu_{1}, \cdots, \mu_{m}\right)
$$

uniformly for $\left(t_{1}, \cdots, t_{m} ; \mu_{1}, \cdots, \mu_{m}\right) \in T^{m} \otimes[-M, M]^{m}$ for every $M>0$. The convergence of Definition 2 is not to be confused with the convergence of distribution functions uniformly in their arguments; this latter concept will not arise in this paper. It is to be noted that the multidimensional continuity theorem implies that uniform convergence in distribution of stochastic processes implies ordinary convergence in distribution thereof.

We next turn to a construction of a specific process sequence:

Definition 3 . The sequence $\left\{x_{n k}, k=1,2, \cdots, k_{n} ; n \geqq 1\right\}$ of finite sequences of independent random variables for which

$$
\lim _{n \rightarrow \infty} \max _{1 \leqq k \leqq k_{n}} p\left\{\left|x_{n k}\right| \geqq \epsilon\right\}=0 \quad \text { for every } \epsilon>0,
$$

'gives rise to a sequence of stochastic processes constructed as follows:

Let

$$
k_{n}(t)=\left[t k_{n}\right]
$$

and let

$$
s_{n k}=\sum_{j=1}^{k} x_{n j}, \quad 1 \leqq k \leqq k_{n}, n \geqq 1
$$

Then let

$$
x_{n}(t, \omega)=s_{n, k_{n}(t)}, \quad 0 \leqq t \leqq 1, n \geqq 1 .
$$

This sequence of stochastic processes with parameter set $[0,1]$ shall be called the sequence $(A)$. If the $x_{n k}$ are identically distributed "within rows," i.e., if $p\left\{x_{n k} \leqq \lambda\right\}$ is independent of $k$, the resulting sequence of stochastic processes shall be called the sequence $(\hat{A})$. We shall write $F_{n k}(\lambda)=p\left\{x_{n k} \leqq \lambda\right\}$, $\phi_{n k}(\mu)=E\left\{\exp i \mu x_{n k}\right\}$.

It is to be observed that the processes of both sequences $(A)$ and $(\hat{A})$ have independent increments. 
We next adopt notations for some convergence criteria upon the sequences $(A)$ and $(\hat{A})$ :

Definition 4. Let $\left\{x_{n}(t, \omega), 0 \leqq t \leqq 1\right\}$ be the sequence $(A)$. Condition (B) will be said to hold if and only if there exist a real function $\gamma(t), 0 \leqq t \leqq 1$, and a bounded function $G(t ; x),-\infty<x<+\infty, t \in[0,1]$, such that

$$
\lim _{n \rightarrow \infty} \sum_{k=1}^{k_{n}(t)}\left(\alpha_{n k}+\int_{-\infty}^{\infty} \frac{x}{1+x^{2}} d F_{n k}\left(x+\alpha_{n k}\right)\right)=\gamma(t), \quad 0 \leqq t \leqq 1
$$

where

$$
\alpha_{n k}=\int_{|x|<\tau} x d F_{n k}(x)=\alpha_{n k}(\tau) \quad \text { for } \tau>0 .
$$

$$
\lim _{n \rightarrow \infty} \sum_{k=1}^{k_{n}(t)} \int_{-\infty}^{x} \frac{u^{2}}{1+u^{2}} d F_{n k}\left(u+\alpha_{n k}\right)=G(t ; x)
$$

at continuity points in $x$ of $G(t, x)$ for each $t \in[0,1]$.

$$
\lim _{n \rightarrow \infty} \sum_{k=1}^{k_{n}(t)} \int_{-\infty}^{\infty} \frac{u^{2}}{1+u^{2}} d F_{n k}\left(u+\alpha_{n k}\right)=G(t ;+\infty), \quad 0 \leqq t \leqq 1 .
$$

Definition 5. Condition (C) will be said to hold for the sequence $(A)$ if and only if there exist a real function $\gamma(t), 0 \leqq t \leqq 1$, and a bounded function $G(t ; x),-\infty<x<+\infty, 0 \leqq t \leqq 1$, such that (a) and (c) of condition (B) hold uniformly for $t \in[0,1]$, and (b) of condition (B) holds, for each $x$ which is a continuity point of $G(1 ; x)$, uniformly for $t \in[0,1]$.

We observe the following obvious properties of $G(t ; x)$ under condition (B):

(i) $G(t ; x)$ is nondecreasing in $(t, x)$ jointly, and non-negative; $G(t ;-\infty)$ $\equiv 0,0 \leqq t \leqq 1$.

(ii) Every continuity point in $x$ of $G(1 ; x)$ is a continuity point in $x$ of $G(t ; x)$ for each $t \in[0,1]$.

(iii) Every continuity point in $t$ of $G(t ;+\infty)$ is a continuity point in $t$ of $G(t ; x)$ for every $x,-\infty<x<+\infty$.

It is, moreover, clear that condition (C) implies condition (B). We shall write, for processes $\left\{x_{n}(t, \omega), t \in T\right\}, n \geqq 1$, and $\{x(t, \omega), t \in T\}$,

$$
\begin{aligned}
\widehat{\phi}_{m}(t, s ; \mu) & =E\left\{\exp i \mu\left(x_{n}(t, \omega)-x_{n}(s, \omega)\right)\right\}, \\
\widehat{\phi}(t, s ; \mu) & =E\{\exp i \mu(x(t, \omega)-x(s, \omega))\} .
\end{aligned}
$$

Finally, for any real $x$ and $\mu$, let

$$
\begin{aligned}
\psi(x, \mu) & =\left(e^{i \mu x}-1-\frac{i \mu x}{1+x^{2}}\right) \frac{1+x^{2}}{x^{2}}, & & x \neq 0 \\
& =-\frac{\mu^{2}}{2}, & & x=0 .
\end{aligned}
$$


Concerning $\psi(x, \mu)$, we observe that

(iv) for any $M>0, \psi(x, \mu)$ is bounded uniformly for $-M \leqq \mu \leqq+M$, $-\infty<x<+\infty$;

(v) for any $a<b, \psi(x, \mu)$ is a continuous function of $x \in[a, b]$ uniformly for $|\mu| \leqq M$ for any $M>0$.

These results are well-known.

2. Convergence of processes with independent increments. We begin with two results simplifying the convergence criteria of Definitions 1 and 2 .

THEOREM 1. Let $\left\{x_{n}(t, \omega), t \in T\right\}$ be a sequence of stochastic processes with independent increments and let $\{x(t, \omega), t \in T\}$ be a stochastic process with independent increments such that

$$
E\{\exp i \mu x(t, \omega)\}
$$

is never zero. Then a necessary and sufficient condition that $\left\{x_{n}(t, \omega), t \in T\right\}$ converge in distribution to $\{x(t, \omega), t \in T\}$ is that for each $t \in T,\left\{x_{n}(t, \omega), n \geqq 1\right\}$ converges in distribution to $x(t, \omega)$.

Proof. The necessity is trivial, and the condition on $E\{\exp i \mu x(t, \omega)\}$ $=\phi(t ; \mu)$ is unnecessary. To show sufficiency it suffices to observe that if $t_{1}, \cdots, t_{m} \in T$, then $\phi_{n}\left(t_{1}, \cdots, t_{m} ; \mu_{1}, \cdots, \mu_{m}\right)$ is a product of quotients of $\phi_{n}\left(t_{k} ; \mu_{j}\right)$ from the hypotheses of independence, and similarly for $\phi\left(t_{1}, \cdots, t_{m}\right.$; $\left.\mu_{1}, \cdots, \mu_{m}\right)$.

Hence

$$
\lim _{n \rightarrow \infty} \phi_{n}(t ; \mu)=\phi(t ; \mu)
$$

for every $t \in T$ and all real $\mu$ implies

$$
\lim _{n \rightarrow \infty} \phi_{n}\left(t_{1}, \cdots, t_{m} ; \mu_{1}, \cdots, \mu_{m}\right)=\phi\left(t_{1}, \cdots, t_{m} ; \mu_{1}, \cdots, \mu_{m}\right)
$$

which, from the multidimensional continuity theorem, implies the criterion of Definition 1.

If the nonvanishing condition on the limiting process fails to hold, we have

THEOREM 2. If $\left\{x_{n}(t, \omega), t \in T\right\}$ is a sequence of stochastic processes with independent increments, and $\{x(t, \omega), t \in T\}$ is a stochastic process with independent increments, a necessary and sufficient condition that $\left\{x_{n}(t, \omega), t \in T\right\}$ converge in distribution to $\{x(t, \omega), t \in T\}$ is that for every $t, s \in T$, the sequence $\left\{\left(x_{n}(t, \omega), x_{n}(s, \omega)\right), n \geqq 1\right\}$ of pairs of random variables converge in distribution to $(x(t, \omega), x(s, \omega))$.

Proof. Again, the necessity is trivial.

Since the convergence in distribution of $\left\{x_{n}(t, \omega), x_{n}(s, \omega), n \geqq 1\right\}$ to $(x(t, \omega), x(s, \omega))$ implies the convergence in distribution of $\left\{x_{n}(t, \omega)-x_{n}(s, \omega)\right.$, $n \geqq 1\}$ to $x(t, \omega)-x(s, \omega)$, we have for all $\mu \lim _{n \rightarrow \infty} E\left\{\exp i \mu\left(x_{n}(t, \omega)-x_{n}(s, \omega)\right)\right\}$ 
$=E\{\exp i \mu(x(t, \omega)-x(s, \omega))\}$. Since, for any $t_{1}, \cdots, t_{m} \in T$, and $k \leqq m$, $t_{1}<t_{2}<\cdots<t_{m}, t_{0}=0$,

$\phi_{n}\left(t_{1}, \cdots, t_{k} ; \mu_{1}, \cdots, \mu_{k}\right)=\phi_{n}\left(t_{1}, \cdots, t_{k-1} ; \mu_{1}, \cdots, \mu_{k-1}+\mu_{k}\right) \widehat{\phi}_{n}\left(t_{k}, t_{k-1} ; \mu_{k}\right)$

and similarly for $\phi\left(t_{1}, \cdots, t_{m} ; \mu_{1}, \cdots, \mu_{m}\right)$, we have

$$
\lim _{n \rightarrow \infty} \phi_{n}\left(t_{1}, \cdots, t_{m} ; \mu_{1}, \cdots, \mu_{m}\right)=\phi\left(t_{1}, \cdots, t_{m} ; \mu_{1}, \cdots, \mu_{m}\right)
$$

by induction. Again, this implies the criterion of Definition 1. Using the method of Theorem 2, one finds the following:

Corollary: Theorem 2. If $\left\{x_{n}(t, \omega), t \in T\right\}$ is a sequence of stochastic processes with independent increments converging in distribution to the stochastic process $\{x(t, \omega), t \in T\}$, then $\{x(t, \omega), t \in T\}$ has independent increments.

Proof. It suffices to observe that for any $t_{1}, \cdots, t_{m} \in T, t_{j}<t_{j+1}$,

$$
\begin{aligned}
E\left\{\operatorname { e x p } i \sum _ { j = 1 } ^ { m } \mu _ { j } \left(x_{n}\left(t_{j}, \omega\right)\right.\right. & \left.\left.-x_{n}\left(t_{j-1}, \omega\right)\right)\right\} \\
& =\phi_{n}\left(t_{1}, \cdots, t_{m} ; \mu_{1}-\mu_{2}, \mu_{2}-\mu_{3}, \cdots, \mu_{m-1}-\mu_{m}, \mu_{m}\right) \\
& =\prod_{j=1}^{m} \widehat{\phi}_{n}\left(t_{j}, t_{j-1} ; \mu_{j}\right)
\end{aligned}
$$

for each $n \geqq 1$. Since the second and third members of this equality converge, respectively, to

$$
\phi\left(t_{1}, \cdots, t_{m} ; \mu_{1}-\mu_{2}, \cdots, \mu_{m}\right)
$$

and

$$
\prod_{j=1}^{m} \widehat{\phi}\left(t_{j} ; t_{j-1} ; \mu_{j}\right)
$$

we have

$$
\begin{aligned}
E\left\{\exp i \sum_{j=1}^{m} \mu_{j}\left(x\left(t_{j}, \omega\right)-x\left(t_{j-1}, \omega\right)\right)\right\} & =\phi\left(t_{1}, \cdots, t_{m} ; \mu_{1}-\mu_{2}, \cdots, \mu_{m}\right) \\
& =\prod_{j=1}^{m} \widehat{\phi}\left(t_{j}, t_{j-1} ; \mu_{j}\right)
\end{aligned}
$$

and the corollary is proved.

For convergence uniformly in distribution, Theorem 1 becomes

THEOREM 3. Let $\left\{x_{n}(t, \omega), t \in T\right\}$ be a sequence of stochastic processes with independent increments, and let $\{x(t, \omega), t \in T\}$ be a stochastic process with independent increments and no fixed points of discontinuity. Let $T$ be compact. Then 
a necessary and sufficient condition that $\left\{x_{n}(t, \omega), t \in T\right\}$ converge uniformly in distribution to $\{x(t, \omega), t \in T\}$ is that

$$
\lim _{n \rightarrow \infty} \phi_{n}(t ; \mu)=\phi(t ; \mu)
$$

uniformly for $(t, \mu) \in T \otimes[-M, M]$ for any $M>0$.

Proof. As before, the necessity is immediate. The sufficiency follows as in Theorem 1, the uniformity conditions of Definition 2 being a consequence of the uniformity condition on $\phi_{n}(t ; \mu) \rightarrow \phi(t ; \mu)$, and the compactness of $T$; the continuity condition insures that $\phi(t ; \mu)$ is continuous in $(t, \mu)$ jointly and infinitely divisible.

3. The sequence $(A)$. We now consider the convergence properties of the sequence $(A)$ of $\$ 1$ under the conditions (B) and (C).

Theorem 4. A necessary and sufficient condition that there exist a stochastic process $\{x(t, \omega), 0 \leqq t \leqq 1\}$ with independent increments to which the sequence $(A)$ converges in distribution is that condition (B) hold. In this case,

$$
\phi(t ; \mu)=\exp \left(i \mu \gamma(t)+\int_{-\infty}^{\infty} \psi(x, \mu) d G(t ; x)\right)
$$

and $\{x(t, \omega), t \in[0,1]\}$ is without fixed points of discontinuity if and only if $\gamma(t)$ and $G(t ;+\infty)$ are continuous functions of $t \in[0,1]$.

Proof. I. Sufficiency. For each $t \in[0,1]$, Gnedenko's theorem applies to $\left\{x_{n k}, k=1, \cdots, k_{n}(t) ; n \geqq 1\right\}$ from condition (B). Hence for each $t$, $\left\{s_{n, k_{n}(t)}, n \geqq 1\right\}$ converges in distribution, and

$$
\phi(t ; \mu)=\exp \left(i \mu \gamma(t)+\int_{-\infty}^{\infty} \psi(x, \mu) d G(t ; x)\right) .
$$

Since the limiting distribution is then infinitely divisible, $\phi(t ; \mu)$ is never zero. Hence if $t>s$,

$$
\begin{aligned}
\widehat{\phi}(t, s ; \mu) & =\frac{\phi(t ; \mu)}{\phi(s ; \mu)} \\
& =\exp \left(i \mu[\gamma(t)-\gamma(s)]+\int_{-\infty}^{\infty} \psi(x, \mu) d[G(t ; x)-G(s ; x)]\right)
\end{aligned}
$$

is always defined, and from properties $1: \mathrm{i}$, ii, iii, $\widehat{\phi}(t, s ; \mu)$ is a characteristic function. Let, for each $m>0$ and $t_{0}=0<t_{1}<\cdots<t_{m} \leqq 1$,

$$
\phi\left(t_{1}, \cdots, t_{m} ; \mu_{1}, \cdots, \mu_{m}\right)=\prod_{k=1}^{m} \widehat{\phi}\left(t_{k}, t_{k-1} ; \sum_{j=k}^{m} \mu_{j}\right) .
$$

The $\phi$ 's so defined clearly generate a consistent family of finite-dimensional characteristic functions, so that there is, from Kolmogorov's Consistency 
Theorem, a stochastic process $\{x(t, \omega), 0 \leqq t \leqq 1\}$ having the $\phi$ 's as their finite-dimensional characteristic functions. From the manner of the definition of the $\phi^{\prime} \mathbf{s},\{x(t, \omega), t \in[0,1]\}$ has independent increments. Since $\left\{x_{n}(t, \omega)\right.$, $n \geqq 1\}$ converges in distribution to $x(t, \omega)$ for each $t \in[0,1]$, again from Gnedenko's Theorem, and $\phi(t ; \mu)$ is never zero, Theorem 1 applies; this proves the sufficiency.

II. Necessity. Assuming $\left\{x_{n}(t, \omega), t \in[0,1]\right\}$ converges in distribution to $\{x(t, \omega), t \in[0,1]\}$, the necessity part of Gnedenko's Theorem implies (a), (b), and (c) of condition (B).

III. If either necessity or sufficiency above holds, then $\left\{x_{n}(t, \omega), t \in[0,1]\right\}$ converges in distribution to $\{x(t, \omega), t \in[0,1]\}$, the limit process having independent increments, and such that

$$
\phi(t ; \mu)=\exp \left(i \mu \gamma(t)+\int_{-\infty}^{\infty} \psi(x, \mu) d G(t ; x)\right) .
$$

If then $\{x(t, \omega), t \in[0,1]\}$ has no fixed points of discontinuity, it follows that

$$
p\left\{\lim _{n \rightarrow \infty} x\left(t_{m}, \omega\right)=x(t, \omega)\right\}=1
$$

for each $t \in[0,1]$ and each sequence $\left\{t_{j}\right\}$ such that

$$
\lim _{m \rightarrow \infty} t_{m}=t, \quad t_{m} \in[0,1] .
$$

Clearly, then, $x\left(t_{m}, \omega\right)$ converges in distribution to $x(t, \omega)$ for any sequence, implying from (1) that

$$
\begin{aligned}
\lim _{m \rightarrow \infty} \gamma\left(t_{m}\right) & =\gamma(t), \\
\lim _{m \rightarrow \infty} G\left(t_{m} ; \infty\right) & =G(t ; \infty)
\end{aligned}
$$

whence $\gamma(t)$ and $G(t ;+\infty)$ are continuous in $t$. If (2) holds, conversely, then $x(t, \omega)-x\left(t_{m}, \omega\right)$ converges in distribution to the unitary random variable, and hence

$$
p\left\{\lim _{m \rightarrow \infty}\left(x(t, \omega)-x\left(t_{m}, \omega\right)\right)=0\right\}=1 .
$$

This completes the proof of the theorem.

The next result relates condition (C) to the uniform convergence in distribution of the sequence $(A)$.

Theorem 5. A sufficient condition that there exist a stochastic process $\{x(t, \omega), t \in[0,1]\}$ with independent increments and no fixed points of discontinuity to which the sequence $(A)$ converges uniformly in distribution is that condition (C) hold. 
Proof. From Theorem 4, there must exist a process $\{x(t, \omega), t \in[0,1]\}$ with independent increments such that $\left\{x_{n}(t, \omega), t \in[0,1]\right\}$ converges in distribution to $\{x(t, \omega), t \in[0,1]\}$. From Theorem 3 , it will suffice to show that condition (C) implies that $\{x(t, \omega), t \in[0,1]\}$ has no fixed points of discontinuity and that $\lim _{n \rightarrow \infty} \phi_{n}(t ; \mu)=\phi(t ; \mu)$ uniformly in $(t, \mu) \in[0,1]$ $\otimes[-M, M]$ for any $M>0$, where, from Theorem 4 ,

$$
\phi(t ; \mu)=\exp \left(i \mu \gamma(t)+\int_{-\infty}^{\infty} \psi(x, \mu) d G(t ; x)\right) .
$$

We first remark that the requirement of infinitesimalness implies

$$
\begin{array}{r}
\lim _{n \rightarrow \infty} \max _{1 \leqq k \leqq k_{n}}\left|\alpha_{n k}\right|=0, \\
\lim _{n \rightarrow \infty} \max _{1 \leqq k \leqq k_{n}}\left|\int_{-\infty}^{\infty} \frac{x^{-\infty}}{1+x^{2}} d F_{n k}\left(x+\alpha_{n k}\right)\right|=0, \\
\lim _{n \rightarrow \infty} \max _{1 \leqq k \leqq k_{n}}\left|\int_{-\infty}^{\infty} \frac{x^{2}}{1+x^{2}} d F_{n k}\left(x+\alpha_{n k}\right)\right|=0
\end{array}
$$

and

$$
\lim _{n \rightarrow \infty} \max _{1 \leqq k \leqq k_{n}}\left|1-\phi_{n k}(\mu)\right|=0
$$

uniformly for $\mu \in[-M, M]$, for any $M>0$. Let

$$
\phi_{n k}(\mu)=e^{-i \mu \alpha_{n k}} \phi_{n k}(\mu)=\int_{-\infty}^{\infty} e^{i \mu x} d F_{n k}\left(x+\alpha_{n k}\right) .
$$

Then

$$
\left|1-\hat{\phi}_{n k}(\mu)\right| \leqq\left|1-\phi_{n k}(\mu)\right|+\left|1-e^{-i \mu \alpha_{n k}}\right|\left|\phi_{n k}(\mu)\right|
$$

implies

$$
\lim _{n \rightarrow \infty} \max _{1 \leqq k \leq k_{n}}\left|1-\widehat{\phi}_{n k}(\mu)\right|=0,
$$

uniformly for $\mu \in[-M, M]$, for any $M>0$. We have also

$$
\begin{aligned}
\mid \sum_{k=1}^{k_{n}(t)}\left(\widehat{\phi}_{n k}(\mu)\right. & -1)-\sum_{k=1}^{k_{n}(t)} \log \widehat{\phi}_{n k}(\mu) \mid \\
& \leqq \frac{1}{4 \pi} \sum_{k=1}^{k_{n}(t)}\left|1-\widehat{\phi}_{n k}(\mu)\right|^{2}\left|\int_{|\zeta|=1 / 2} \frac{\log (1-\zeta) d \zeta}{\zeta^{3}\left(\zeta-\left(-\widehat{\phi}_{n k}(\mu)\right)\right)}\right| \\
& \leqq D \max _{1 \leqq k \leqq k_{n}}\left|1-\widehat{\phi}_{n k}(\mu)\right| \sum_{k=1}^{k_{n}}\left|1-\widehat{\phi}_{n k}(\mu)\right| \\
& \leqq D \max _{1 \leq t \leq t_{n}}\left|1-\widehat{\phi}_{n k}(\mu)\right|\left(-\log \left|\phi_{n}(1 ; \mu)\right|\right)
\end{aligned}
$$


where $D$ is independent of $k, n$, and $\mu$ for large $n$. Hence

$$
\lim _{n \rightarrow \infty}\left(\sum_{k=1}^{k_{n}(t)}\left(\widehat{\phi}_{n k}(\mu)-1\right)-\sum_{k=1}^{k_{n}(t)} \log \widehat{\phi}_{n k}(\mu)\right)=0
$$

uniformly for $(t, \mu) \in[0,1] \otimes[-M, M]$ for any $M>0$. We next observe that

$$
\lim _{n \rightarrow \infty} \sum_{k=1}^{k_{n}(t)} \int_{-\infty}^{\infty} \psi(x, \mu) \frac{x^{2}}{1+x^{2}} d F_{n k}\left(x+\alpha_{n k}\right)=\int_{-\infty}^{\infty} \psi(x ; \mu) d G(t ; x)
$$

uniformly for $(t, \mu) \in[0,1] \otimes[-M, M]$, since $\psi(x, \mu)$ is bounded and continuous in $x$, uniformly for $\mu \in[-M, M]$, and

$$
\lim _{n \rightarrow \infty} \int_{-\infty}^{x} \frac{u^{2}}{1+u^{2}} d F_{n k}\left(u+\alpha_{n k}\right)=G(t ; x)
$$

uniformly for $t \in[0,1]$ at continuity points of $G(1, x)$. The proof is a step-bystep reproduction of the Helly-Bray Theorem, and the details will be omitted.

Using (2), it is to be observed that since

$$
\begin{aligned}
& \left|\sum_{k=1}^{k_{n}(t)}\left(\widehat{\phi}_{n k}(\mu)-1\right)+i \mu \sum_{k=1}^{k_{n}(t)} \alpha_{n k}-\log \phi(t ; \mu)\right| \\
& \leqq|\mu|\left|\sum_{k=1}^{k_{n}(t)}\left(\alpha_{n k}+\int_{-\infty}^{\infty} \frac{x}{1+x^{2}} d F_{n k}\left(x+\alpha_{n k}\right)\right)-\gamma(t)\right| \\
& \quad+\left|\sum_{k=1}^{k_{n}(t)} \int_{-\infty}^{\infty} \psi(x, \mu) \frac{x^{2}}{1+x^{2}} d F_{n k}\left(x+\alpha_{n k}\right)-\int_{-\infty}^{\infty} \psi(x, \mu) d G(t ; x)\right|
\end{aligned}
$$

we must have

$$
\lim _{n \rightarrow \infty} \sum_{k=1}^{k_{n}(t)}\left(\widehat{\phi}_{n k}(\mu)-1\right)+i \mu \sum_{k=1}^{k_{n}(t)} \alpha_{n k}-\log \phi(t ; \mu)=0
$$

uniformly for $(t, \mu) \in[-M, M]$ for any $M>0$, from (a) of condition (C). Finally, we obtain

$$
\begin{aligned}
\left|\log \phi_{n}(t ; \mu)-\log \phi(t ; \mu)\right| \leqq & \left|\sum_{k=1}^{k_{n}(t)}\left(\widehat{\phi}_{n k}(\mu)-1\right)+i \mu \sum_{k=1}^{k_{n}(t)} \alpha_{n k}-\log \phi(t ; \mu)\right| \\
& +\left|\sum_{k=1}^{k_{n}(t)} \log \widehat{\phi}_{n k}(\mu)-\sum_{k=1}^{k_{n}(t)}\left(\widehat{\phi}_{n k}(\mu)-1\right)\right|
\end{aligned}
$$

and from (1) and (3) we conclude

$$
\lim _{n \rightarrow \infty} \log \phi_{n}(t ; \mu)=\log \phi(t ; \mu), \text { uniformly for }(t, \mu) \in[0,1] \otimes[-M, M]
$$

which implies

$$
\lim _{n \rightarrow \infty} \phi_{n}(t ; \mu)=\phi(t ; \mu)
$$


uniformly for $(t, \mu) \in[0,1] \otimes[-M, M]$ for any $M>0$, since the limit is bounded uniformly (in $t$ and $\mu$ ) away from zero. From Theorem 3 , the uniform convergence in distribution of $\left\{x_{n}(t, \omega), t \in[0,1]\right\}$ to $\{x(t, \omega), t \in[0,1]\}$ is established.

To show that the limiting process has no fixed points of discontinuity, it will suffice to show that $\gamma(t)$ and $G(t ;+\infty)$ (and hence also $G(t ; x)$ at continuity points of $G(1 ; x))$ are continuous functions of $t \in[0,1]$. Choose $\epsilon>0$, and let $N_{1}$ be so large that $n \geqq N_{1} \Rightarrow$

$$
\max _{1 \leqq k \leqq k_{n}}\left|\int_{-\infty}^{\infty} \frac{x}{1+x^{2}} d F_{n k}\left(x+\alpha_{n k}\right)\right|<\frac{\epsilon}{6}
$$

and

$$
\max _{1 \leqq k \leqq k_{n}}\left|\alpha_{n k}\right|<\frac{\epsilon}{6} \text {. }
$$

Then $\exists N_{2} \geqq N_{1} \ni n \geqq N_{2} \Rightarrow$ for all $t \in[0,1]$,

$$
\left|\gamma(t)-\sum_{k=1}^{k_{n}(t)}\left(\alpha_{n k}+\int_{-\infty}^{\infty} \frac{x}{1+x^{2}} d F_{n k}\left(x+\alpha_{n k}\right)\right)\right|<\frac{\epsilon}{3} .
$$

Let $\delta \leqq 1 / 2 k_{N_{2}}$. Then if $t \geqq s, 0<t-s<\delta \Rightarrow$

$$
\begin{aligned}
|\gamma(t)-\gamma(s)| \leqq & \left|\gamma(t)-\sum_{k=1}^{k_{N_{2}}(t)}\left(\alpha_{N_{2} k}+\int_{-\infty}^{\infty} \frac{x}{1+x^{2}} d F_{N_{2} k}\left(x+\alpha_{N_{2} k}\right)\right)\right| \\
& +\left|\sum_{k=k_{N_{2}(s)+1}}^{k_{N_{2}(t)}}\left(\alpha_{N_{2} k}+\int_{-\infty}^{\infty} \frac{x}{1+x^{2}} d F_{N_{2} k}\left(x+\alpha_{N_{2} k}\right)\right)\right| \\
& +\left|\gamma(s)-\sum_{k=1}^{k_{N_{2}(s)}}\left(\alpha_{N_{2} k}+\int_{-\infty}^{\infty} \frac{x}{1+x^{2}} d F_{N_{2} k}\left(x+\alpha_{N_{2} k}\right)\right)\right| \\
< & \frac{\epsilon}{3}+\frac{\epsilon}{3}+\frac{\epsilon}{3}=\epsilon
\end{aligned}
$$

since the sum

$$
\sum_{k=k_{N_{2}}(s)+1}^{k_{N_{2}}(t)}
$$

contains at most one term, this term being bounded by

$$
\begin{gathered}
\max _{1 \leqq k \leqq k_{N_{2}}}\left|\alpha_{N_{2} k}\right|+\max _{1 \leqq k \leqq k_{N_{2}}} \mid \int_{-\infty}^{\infty} \frac{x}{1+x^{2}} d F_{N_{2} k}\left(x+\alpha_{N_{2} k} \mid\right. \\
<\frac{\epsilon}{6}+\frac{\epsilon}{6}=\frac{\epsilon}{3} .
\end{gathered}
$$


Hence, $\gamma(t)$ is a continuous function of $t \in[0,1]$.

For any $\epsilon>0$ we can also find $N_{3} \ni n \geqq N_{3} \Rightarrow$

$$
\max _{1 \leqq k \leqq k_{n}} \int_{-\infty}^{\infty} \frac{x^{2}}{1+x^{2}} d F_{n k}\left(x+\alpha_{n k}\right)<\frac{\epsilon}{3}
$$

and $N_{4} \geqq N_{3} \ni n \geqq N_{4} \Rightarrow$

$$
\left|G(t ;+\infty)-\sum_{k=1}^{k_{n}(t)} \int_{-\infty}^{\infty} \frac{x^{2}}{1+x^{2}} d F_{n k}\left(x+\alpha_{n k}\right)\right|<\frac{\epsilon}{3} .
$$

Let $\delta \leqq 1 / 2 k_{N_{\triangleleft}}$; then if $0<t-s<\delta, 0 \leqq t, s \leqq 1$, we have

$$
\begin{aligned}
\mid G(t ;+\infty)-G(s ;+\infty \mid \leqq & \left|G(t ;+\infty)-\sum_{k=1}^{k_{N_{4}}(t)} \int_{-\infty}^{\infty} \frac{x^{2}}{1+x^{2}} d F_{N_{4} k}\left(x+\alpha_{N_{4} k}\right)\right| \\
& +\left|\sum_{k=k_{N_{4}(s)+1}}^{k_{N_{4}(t)}} \frac{x^{2}}{1+x^{2}} d F_{N_{4} k}\left(x+\alpha_{N_{4} k}\right)\right| \\
& +\left|G(s ;+\infty)-\sum_{k=1}^{k_{N_{4}(s)}} \int_{-\infty}^{\infty} \frac{x^{2}}{1+x^{2}} d F_{N_{4} k}\left(x+\alpha_{N_{4} k}\right)\right| \\
< & \frac{\epsilon}{3}+\frac{\epsilon}{3}+\frac{\epsilon}{3}=\epsilon
\end{aligned}
$$

and $G(t ;+\infty)$ is a continuous function of $t \in[0.1]$.

This completes the proof of Theorem 5 .

Theorem 5 can be considerably strengthened if the random variables $\left\{x_{n k}, 1 \leqq k \leqq k_{n}, n \geqq 1\right\}$ are identically distributed for each $n$ :

THEOREM 6. A necessary and sufficient condition that there exist a stochastic process $\{x(t, \omega), t \in[0,1]\}$ with independent increments to which the sequence $(\hat{A})$ converges in distribution is that the sequence of random variables $\left\{x_{n}(1, \omega)\right.$, $n \geqq 1\}$ converge in distribution. Moreover, in this case the sequence $(\hat{A})$ converges uniformly in distribution to $\{x(t, \omega) ; t \in[0,1]\}$ and the limiting process has stationary increments and is without fixed points of discontinuity.

Proof. The necessity is trivial. Let

$$
F_{n}(x)=F_{n k}(x), \quad \alpha_{n}=\alpha_{n k} .
$$

Since $x_{n}(1, \omega)=s_{n, k_{n}}$, Gnedenko's Theorem (necessity) asserts that if $\left\{s_{n, k_{n}}\right.$, $n \geqq 1\}$ converges in distribution, then there exist a constant $\gamma$ and a function $G(x)$, bounded and nondecreasing, such that

$$
\begin{aligned}
& \lim _{n \rightarrow \infty} \sum_{k=1}^{k_{n}}\left(\alpha_{n k}+\int_{-\infty}^{\infty} \frac{x}{1+x^{2}} d F_{n k}\left(x+\alpha_{n k}\right)\right) \\
&=\lim _{n \rightarrow \infty} k_{n}\left(\alpha_{n}+\int_{-\infty}^{\infty} \frac{x}{1+x^{2}} d F_{n}\left(x+\alpha_{n}\right)\right)=\gamma,
\end{aligned}
$$




$$
\lim _{n \rightarrow \infty} \sum_{k=1}^{k_{n}} \int_{-\infty}^{x} \frac{u^{2}}{1+u^{2}} d F_{n k}\left(u+\alpha_{n k}\right)=\lim _{n \rightarrow \infty} k_{n} \int_{-\infty}^{x} \frac{u^{2}}{1+u^{2}} d F_{n}\left(u+\alpha_{n}\right)=G(x)
$$

at continuity points of $G(x)$, and

$$
\begin{aligned}
\lim _{n \rightarrow \infty} \sum_{k=1}^{k_{n}} \int_{-\infty}^{\infty} \frac{x^{2}}{1+x^{2}} d F_{n k}\left(x+d_{n k}\right) \\
\quad=\lim _{n \rightarrow \infty} k_{n} \int_{-\infty}^{\infty} \frac{x^{2}}{1+x^{2}} d F_{n}\left(x+\alpha_{n}\right)=G(+\infty) .
\end{aligned}
$$

Let $G(t ; x)=t G(x), 0 \leqq t \leqq 1$, and $\gamma(t)=t \gamma, 0 \leqq t \leqq 1$. Since

$$
\begin{aligned}
\sum_{k=1}^{k_{n}(t)}\left(\alpha_{n k}+\int_{-\infty}^{\infty} \frac{x}{1+x^{2}} d F_{n k}(x\right. & \left.\left.+\alpha_{n k}\right)\right) \\
& =\frac{k_{n}(t)}{k_{n}}\left[k_{n}\left(\alpha_{n}+\int_{-\infty}^{\infty} \frac{x}{1+x^{2}} d F_{n}\left(x+\alpha_{n}\right)\right)\right], \\
\sum_{k=1}^{k_{n}(t)} \int_{-\infty}^{x} \frac{u^{2}}{1+u^{2}} d F_{n k}\left(u+\alpha_{n k}\right) & =\frac{k_{n}(t)}{k_{n}}\left[k_{n} \int_{-\infty}^{x} \frac{u^{2}}{1+u} d F_{n}\left(u+\alpha_{n}\right)\right], \\
\sum_{k=1}^{k_{n}(t)} \int_{-\infty}^{\infty} \frac{x^{2}}{1+x^{2}} d F_{n k}\left(x+\alpha_{n k}\right) & =\frac{k_{n}(t)}{k_{n}}\left[k_{n} \int_{-\infty}^{\infty} \frac{x^{2}}{1+x^{2}} d F_{n}\left(x+\alpha_{n}\right)\right]
\end{aligned}
$$

and since $\lim _{n \rightarrow \infty} k_{n}(t) / k_{n}=t$ uniformly for $t \in[0,1]$, it is clear that condition (C) holds. Applying Theorem 4, it remains only to show that the limiting process has stationary increments. Since, for $t \geqq s$,

$$
\begin{aligned}
E\{\exp i \mu(x(t, \omega)-x(s, \omega))\} & =\phi(t ; \mu) / \phi(s ; \mu) \\
& =\exp \left(i \mu(t-s) \gamma+(t-s) \int_{-\infty}^{\infty} \psi(x, \mu) d G(x)\right) \\
& =[\phi(1 ; \mu)]^{(t-s)}
\end{aligned}
$$

this last conclusion follows also.

It is to be observed that a special case of Theorem 5 is the "cumulative sum" situation; briefly, if $\left\{y_{k}, k \geqq 1\right\}$ is a sequence of independent identically distributed random variables, such that there is a sequence of constants $\left\{A_{n}, n \geqq 1\right\}$ for which $\lim _{n \rightarrow \infty} A_{n}=+\infty$ and the random variables $\left\{\left(1 / A_{n}\right) \sum_{k=1}^{n} y_{k}, n \geqq 1\right\}$ converge in distribution, then Theorem 6 applies, writing

$$
x_{n k}=\frac{1}{A_{n}} y_{k}, \quad 1 \leqq k \leqq n=k_{n}, n \geqq 1 .
$$

This problem has been discussed by several authors (see references), in the process of investigating the ideas of the next section. 
4. The convergence of functionals defined on convergent processsequences. Let $\left\{x_{n}(t, \omega), t \in[0,1]\right\}$ be a sequence of stochastic processes, separable and with independent increments, converging uniformly in distribution to a stochastic process $\{x(t, \omega), t \in[0,1]\}$ which is separable, with independent increments and no fixed points of discontinuity. Let $S$ denote the union of the separability sequences of $\left\{x_{n}(t, \omega), t \in[0,1]\right\}$ for all $n$ and the rationals on $[0,1]$. Since $S$ is then dense in $[0,1]$, it is a separability sequence for $\{x(t, \omega) t \in[0,1]\}$. For each positive integer $N$ we can extract a sequence of finite sequences from $S$, which we denote by $\left\{S_{n k}^{(N)}, 1 \leqq k \leqq N 2^{n}, n=1\right.$, $2, \cdots\}$, such that

$$
\begin{gathered}
\frac{j}{N}=S_{n, j 2^{n}}^{(N)}<S_{n, j 2^{n}+1}^{(N)}<\cdots<S_{n,(j+1) 2^{n}}^{(N)}=\frac{j+1}{N}, 0 \leqq j \leqq N-1 ; \\
S_{n+m, j 2^{2}}^{(N)}=S_{n j}^{(N)}, \quad 1 \leqq j \leqq N 2^{n}, m, n \geqq 1 ; \\
\max _{1 \leqq j \leqq N 2^{n}}\left(S_{n j}^{(N)}-S_{n, j-1}^{(N)}\right)<\frac{1}{N 2^{n-1}} ; \\
\bigcup_{n=1}^{\infty} \bigcup_{j=1}^{N 2^{n}}\left\{S_{n j}^{(N)}\right\}=S .
\end{gathered}
$$

The next result concerns the convergence in distribution of a simple type functional defined on the sample functions of the convergent process-sequence under consideration.

TheOREM 7. Let $N \geqq 1$ be a fixed positive integer, and let $\alpha_{1}, \cdots, \alpha_{N}$ and $\beta_{1}, \cdots, \beta_{N}, \alpha_{j} \leqq \beta_{j}, 1 \leqq j \leqq N$, be continuity vectors of

$$
p\left\{\alpha_{j} \leqq x(t, \omega) \leqq \beta_{j}, \frac{j-1}{N}<t \leqq \frac{j}{N}, 1 \leqq j \leqq N\right\} .
$$

Then

$$
\begin{gathered}
\lim _{n \rightarrow \infty} p\left\{\alpha_{j} \leqq x_{n}(t, \omega) \leqq \beta_{j}, \frac{j-1}{N}<t \leqq \frac{j}{N}, 1 \leqq j \leqq N\right\} \\
=p\left\{\alpha_{j} \leqq x(t, \omega) \leqq \beta_{j}, \frac{j-1}{N}<t \leqq \frac{j}{N}, 1 \leqq j \leqq N\right\}
\end{gathered}
$$

Proof. The elements of the above limit statement are all defined, from the separability conditions. To simplify the notation, let

$$
\begin{aligned}
E_{m}^{(n)}= & \left\{\alpha_{j} \leqq x_{n}\left(S_{m,(j-1) 2^{m}+k}^{(N)}, \omega\right) \leqq \beta_{j}, k=1, \cdots, 2^{m}, j=1, \cdots, N\right\}, \\
E_{m r}^{(n)}= & \left\{x_{n}\left(S_{m j}^{(N)}, \omega\right) \text { satisfies the conditions defining } E_{m}^{(n)} \text { for } j=1,2, \cdots,\right. \\
& \left.r-1, \text { but } x_{n}\left(S_{m r}^{(N)}, \omega\right) \text { fails }\right\} .
\end{aligned}
$$


Let $\epsilon_{0}>0$ be chosen arbitrarily. Choose for each positive integer $p$ an $\epsilon_{p}>0$ so that

$$
\frac{\epsilon_{0}}{2} \leqq \epsilon_{p} \leqq \epsilon_{0}
$$

and

$$
\left\{\alpha_{1} \pm \epsilon_{p}, \cdots, \alpha_{N} \pm \epsilon_{p}, \beta_{1} \pm \epsilon_{p}, \cdots, \beta_{N} \pm \epsilon_{p}\right\}
$$

are continuity vectors of

$$
p\left\{\alpha_{j} \leqq x\left(S_{p,(j-1) 2^{p}+m}^{(N)}, \omega\right) \leqq \beta_{j}, 1 \leqq m \leqq 2^{p}, 1 \leqq j \leqq N\right\} .
$$

Let

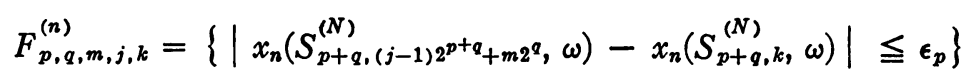

and let

$$
\hat{E}_{p}^{(n)}=\left\{\alpha_{j}+\epsilon_{p} \leqq x_{n}\left(S_{p,(j-1) 2^{p}+m}^{(N)}, \omega\right) \leqq \beta_{j}-\epsilon_{p}, 1 \leqq m \leqq 2^{p}, 1 \leqq j \leqq N\right\} .
$$

Then

$$
\begin{aligned}
1-p\left\{E_{p+q}^{(n)}\right\}= & \sum_{r=1}^{N 2^{p+q}} p\left\{E_{p+q, r}^{(n)}\right\} \\
= & \sum_{j=1}^{N} \sum_{m=1}^{2^{p}} \sum_{k=(j-1) 2^{p+q_{+}}+(m-1) 2^{q}+1}^{(j-1) 2^{p+q}+m^{2 q}} p\left\{E_{p+q, k}^{(n)} \cap F_{p, q, m, j, k}^{(n)}\right\} \\
& \quad+\sum_{j=1}^{N} \sum_{m=1}^{2^{p}} \sum_{k=(j-1) 2^{p+p+(m-1) 2^{q}+1}}^{(j-1) 2^{p+q}+m 2^{q}} P\left\{E_{p+q, k}^{(n)}-F_{p, q, m, j, k}^{(n)}\right\} .
\end{aligned}
$$

Since $E_{p+\varepsilon, r}^{(n)}$ and $F_{p, q, m, j, k}^{(n)}$ are independent, the first sum in the last member can be estimated by the product of the largest of $p\left\{F_{p, q, m, j, k}^{(n)}\right\}$ and a sum of probabilities of disjoint sets, which latter is certainly less than one. The second sum is also a sum of probabilities of disjoint sets, and the two sums can be estimated as follows:

$$
\begin{aligned}
1-p\left\{E_{p+q}^{(n)}\right\} \leqq & \max _{(j-1) 2^{p+q}+(m-1) 2^{q}+1 \leqq k \leqq(j-1)^{2^{p+q}+m 2^{q} ;}} p\left\{F_{p, q, m, j, k}^{(n)}\right\} \\
& +p\left\{\bigcup_{j=1}^{N} \bigcup_{m=1}^{2^{p}} \bigcup_{k=(j-1) 2^{p+q}+(m-1) 2^{q}+1}^{(j-1) 2^{p+q}+m 2^{q}}\left(E_{p+q, k}^{(n)}-F_{p, q, m, j, k}^{(n)}\right) .\right.
\end{aligned}
$$

To estimate the first term of this last expression, we observe that for any random variable $y(\omega)$, 


$$
p\{|y(\omega)| \geqq \epsilon\} \leqq \frac{(M+2 \pi / \epsilon)^{2}}{M^{3}} \int_{0}^{M} R(1-E\{\exp i \mu y\}) d \mu .
$$

Since, however, $\{x(t, \omega), t \in[0,1]\}$ is without fixed points of discontinuity,

$$
\lim _{t \rightarrow \Omega} \phi(t ; \mu)=\phi(s ; \mu)
$$

uniformly for $(s, \mu) \in[0,1] \otimes[-M, M]$ for any $M>0$. Moreover, $\phi(s, \mu)$ is never zero on $[0,1] \otimes[-M, M]$. Hence

$$
\lim _{t \downarrow s} \widehat{\phi}(t, s ; \mu)=\lim _{t \downarrow s} \frac{\phi(t ; \mu)}{\phi(s ; \mu)}=1
$$

uniformly for $(s, \mu) \in[0,1] \otimes[-M, M]$. But also

$$
\lim _{n \rightarrow \infty} \phi_{n}(t ; \mu)=\phi(t ; \mu)
$$

uniformly for $(t, \mu) \in[0,1] \otimes[-M, M]$. Hence,

$$
\lim _{n \rightarrow \infty ; t \downarrow s} \widehat{\phi}_{n}(t, s ; \mu)=1
$$

uniformly for $(s, \mu) \in[0,1] \otimes[-M, M]$. Hence, given $\eta>0$ arbitrarily, there exist $N(\eta)$ and $\delta(\eta)$ such that $n \geqq N(\eta)$ and $0<t-s<\delta(\eta),|\mu| \leqq M \Rightarrow$

$$
\left|1-\widehat{\phi}_{n}(t, s ; \mu)\right|<\eta \text {. }
$$

Thus, for such $n, t, s$, and $\mu$, if $k(\epsilon)=(M+2 \pi / \epsilon)^{2} / M^{2}$,

$$
\begin{aligned}
p\left\{\left|x_{n}(t, \omega)-x_{n}(s, \omega)\right| \geqq \epsilon\right\} & \leqq \frac{\left(M+(2 \pi / \epsilon)^{2}\right.}{M^{3}} \int_{0}^{M} R\left(1-\widehat{\phi}_{n}(t, s ; \mu)\right) d \mu \\
& <k(\epsilon) \frac{1}{M} \int_{0}^{M}\left|1-\widehat{\phi}_{n}(t, s ; \mu)\right| d \mu \\
& \leqq k(\epsilon) \frac{1}{M} \int_{0}^{M} \eta d \mu=k(\epsilon) \eta .
\end{aligned}
$$

We have, therefore, for $n \geqq N(\eta), p>-\log _{2} \delta(\eta)+1$, that

$$
0 \leqq S_{p,(j-1) 2^{p}+m}^{(N)}-S_{p+q, k}^{(N)} \leqq S_{p,(j-1) 2^{p}+m}^{(N)}-S_{p(j-1) 2^{p}+m-1}^{(N)}<\frac{1}{N 2^{p-1}}<\delta(\eta)
$$

and hence

$$
p\left\{F_{p, q, m, j, k}^{(n)}\right\}<k\left(\epsilon_{p}\right) \eta \text { for all } q, m, j \text {, and } k \text {. }
$$

Further,

$$
\bigcup_{j=1}^{N} \bigcup_{m=1}^{2^{p}} \underset{k=(j-1) 2^{p-q_{+(m-1) 2^{q}+1}}}{(j-1) 2^{p+q}+m 2^{q}}\left(E_{p+q, k}^{(n)}-F_{p, q, m, j, k}^{(n)}\right) \subset\left(E_{p}^{(n)}\right)^{-1}
$$


so that, finally, we have

or,

$$
1-p\left\{E_{p+q}^{(n)}\right\}<k\left(\epsilon_{p}\right) \eta+1-p\left\{\hat{E}_{p}^{(n)}\right\}
$$

$$
p\left\{\hat{E}_{p}^{(n)}\right\}<p\left\{E_{p+q}^{(n)}\right\}+k\left(\epsilon_{p}\right) \eta \leqq p\left\{E_{p}^{(n)}\right\}+k\left(\epsilon_{p}\right) \eta .
$$

From property (iv) of the $\left\{S_{n k}^{(N)}\right\}$ and the separability of the $\left\{x_{n}(t, \omega)\right.$, $t \in[0,1]\}$ process, we have

$$
\lim _{a \rightarrow \infty} p\left\{E_{p+q}^{(n)}\right\}=p\left\{\alpha_{j} \leqq x_{n}(t, \omega) \leqq \beta_{j}, \frac{j-1}{N}<t \leqq \frac{j}{N}, 1 \leqq j \leqq N\right\}
$$

Thus, since the extreme members of (1) are independent of $q$, we have

$$
\begin{aligned}
p\left\{\hat{E}_{p}^{(n)}\right\} & \leqq p\left\{\alpha_{j} \leqq x_{n}(t, \omega) \leqq \beta_{j}, \frac{j-1}{N}<t \leqq \frac{j}{N}, 1 \leqq j \leqq N\right\}+\eta k\left(\epsilon_{p}\right) \\
& \leqq p\left\{E_{p}^{(n)}\right\}+k\left(\epsilon_{p}\right) \eta .
\end{aligned}
$$

Let $k_{0}=\left(1 / M^{2}\right)\left(M+4 \pi / \epsilon_{0}\right)^{2}$; then $k\left(\epsilon_{p}\right) \leqq k_{0}$ for all $p$, and

$$
\begin{aligned}
p\left\{\hat{E}_{p}^{(n)}\right\} & \leqq p\left\{\alpha_{j} \leqq x_{n}(t, \omega) \leqq \beta_{j}, \frac{j-1}{N}<t \leqq \frac{j}{N}, 1 \leqq j \leqq N\right\}+\eta k_{0} \\
& \leqq p\left\{E_{p}^{(n)}\right\}+k_{0} \eta .
\end{aligned}
$$

Moreover,

$$
\begin{array}{r}
\lim _{n \rightarrow \infty} p\left\{\hat{E}_{p}^{(n)}\right\}=p\left\{\alpha_{j}+\epsilon_{p} \leqq x\left(S_{p,(j-1) 2^{p}+m}^{(N)}, \omega\right) \leqq \beta_{j}-\epsilon_{p},\right. \\
\left.1 \leqq m \leqq 2^{p}, 1 \leqq j \leqq N\right\}
\end{array}
$$

and similarly for $p\left\{E_{p}^{(n)}\right\}$, since the $x_{n}(t, \omega), t \in[0,1]$ converge in distribution to $x(t, \omega), t \in[0,1]$. Hence,

$$
\begin{aligned}
p\left\{\alpha_{j}+\epsilon_{p}\right. & \left.\leqq x\left(S_{p,(j-1) 2^{p}+m}^{(N)}, \omega\right) \leqq \beta_{j}-\epsilon_{p}, 1 \leqq m \leqq 2^{p}, 1 \leqq j \leqq N\right\} \\
& \leqq p\left\{\alpha_{j} \leqq x_{r}(t, \omega) \leqq \beta_{j}, \frac{j-1}{N}<t \leqq \frac{j}{N}, 1 \leqq j \leqq N\right\}+\eta k_{0}
\end{aligned}
$$

and since $\epsilon_{p} \leqq \epsilon_{0}$, we finally have

$$
\begin{aligned}
p\left\{\alpha_{j}+\epsilon_{0}\right. & \left.\leqq x\left(S_{p,(j-1) 2^{p}+m}^{(N)}, \omega\right) \leqq \beta_{j}-\epsilon_{0}, 1 \leqq m \leqq 2^{p}, 1 \leqq j \leqq N\right\} \\
& \leqq p\left\{\alpha_{j} \leqq x_{n}(t, \omega) \leqq \beta_{j}, \frac{j-1}{N}<t \leqq \frac{j}{N}, 1 \leqq j \leqq N\right\}+\eta k_{0} \\
& \leqq p\left\{\alpha_{j} \leqq x\left(S_{p,(j-1) 2^{p}+m}^{(N)}, \omega\right) \leqq \beta_{j}, 1 \leqq m \leqq 2^{p}, 1 \leqq j \leqq N\right\}+\eta k_{0}
\end{aligned}
$$

for $p>-\log _{2} \delta(\eta)+1, n \geqq N(\eta)$. Passing to the limits superior and inferior as 
$n \rightarrow \infty$ on the middle member, and the limits as $p \rightarrow \infty$ on the extreme members, we have, since $\eta>0$ was arbitrary,

$$
\begin{aligned}
\lim _{p \rightarrow \infty} p\left\{\alpha_{j}+\right. & \left.\epsilon_{0} \leqq x\left(S_{p,(j-1) 2^{p}+m}^{(N)}, \omega\right) \leqq \beta_{j}-\epsilon_{0}, 1 \leqq m \leqq 2^{p}, 1 \leqq j \leqq N\right\} \\
& =p\left\{\alpha_{j}+\epsilon_{0} \leqq x(t, \omega) \leqq \beta_{j}-\epsilon_{0}, \frac{j-1}{N}<t \leqq \frac{j}{N}, 1 \leqq j \leqq N\right\} \\
& \leqq \liminf _{n \rightarrow \infty} p\left\{\alpha_{j} \leqq x_{n}(t, \omega) \leqq \beta_{j}, \frac{j-1}{N}<t \leqq \frac{j}{N}, 1 \leqq j \leqq N\right\} \\
& \leqq \limsup _{n \rightarrow \infty} p\left\{\alpha_{j} \leqq x_{n}(t, \omega) \leqq \beta_{j}, \frac{j-1}{N}<t \leqq \frac{j}{N}, 1 \leqq j \leqq N\right\} \\
& \leqq \lim _{p \rightarrow \infty} p\left\{\alpha_{j} \leqq x\left(S_{p,(j-1) 2^{p}+m}^{(N)}, \omega\right) \leqq \beta_{j}, 1 \leqq m \leqq 2^{p}, 1 \leqq j \leqq N\right\} \\
& =p\left\{\alpha_{j} \leqq x(t, \omega) \leqq \beta_{j}, \frac{j-1}{N}<t \leqq \frac{j}{N}, 1 \leqq j \leqq N\right\}
\end{aligned}
$$

using the separability of the $\{x(t, \omega), t \in[0,1]\}$ process in finding the limits on the extreme members. Since

$$
\begin{aligned}
& \lim _{\epsilon_{0} \rightarrow 0} p\left\{\alpha_{j}+\epsilon_{0} \leqq x(t, \omega) \leqq \beta_{j}-\epsilon_{0}, \frac{j-1}{N}<t \leqq \frac{\jmath}{N}, 1 \leqq j \leqq N\right\} \\
& =p\left\{\alpha_{j} \leqq x(t, \omega) \leqq \beta_{j}, \frac{j-1}{N}<t \leqq \frac{j}{N}, 1 \leqq j \leqq N\right\}
\end{aligned}
$$

the theorem is proved.

Theorem 7. Corollary. If $\lambda$ is a continuity point of $p\left\{\sup _{0 \leqq t \leqq 1} x(t, \omega)\right.$ $\leqq \lambda\}$, then

$$
\lim _{n \rightarrow \infty} p\left\{\sup _{0 \leqq t \leqq 1} x_{n}(t, \omega) \leqq \lambda\right\}=p\left\{\sup _{0 \leqq t \leqq 1} x(t, \omega) \leqq \lambda\right\} .
$$

Proof. We remark that if $x \geqq \alpha_{j}+\epsilon$ is interpreted to mean $x \geqq-1 / \epsilon$, the above proof carries through, step by step, when $N=1$ and $\alpha_{1}=-\infty$. Set $\beta_{1}=\lambda$, and the corollary follows.

Let, for each positive integer $n$,

$$
\begin{aligned}
& \sigma_{n j}^{(N)}(\omega)=\inf _{(j-1) / N<t \leqq j / N} x_{n}(t, \omega), \rho_{n j}^{(N)}(\omega)=\sup _{(j-1) / N<t \leqq j / N} x_{n}(t, \omega) \\
& \sigma_{j}^{(N)}(\omega)=\inf _{(j-1) / N<t \leqq j / N} x(t, \omega), \rho_{j}^{(N)}(\omega)=\sup _{(j-1) / N<t \leqq j / N} x(t, \omega) . \quad 1 \leqq j \leqq N,
\end{aligned}
$$


Then Theorem 7 states that $\left\{\rho_{n 1}^{(N)}, \cdots, \rho_{n N}^{(N)}, \sigma_{n 1}^{(N)}, \cdots, \sigma_{n N}^{(N)}\right\}$ converges in distribution to $\left\{\rho_{1}^{(N)}, \cdots, \rho_{N}^{(N)}, \sigma_{1}^{(N)}, \cdots, \sigma_{N}^{(N)}\right\}$. The next theorem extends this result:

TheOREM 8. If $f\left(\xi_{1}, \cdots, \xi_{2 N}\right)$ is a bounded continuous function on $2 N$-dimensional Euclidean space, then

$$
\begin{aligned}
\lim _{n \rightarrow \infty} & E\left\{f\left(\rho_{n 1}^{(N)}, \cdots, \rho_{n N}^{(N)}, \sigma_{n 1}^{(N)}, \cdots, \sigma_{n N}^{(N)}\right)\right. \\
= & \left\{f\left(\rho_{1}^{(N)}, \cdots, \rho_{N}^{(N)}, \sigma_{1}^{(N)}, \cdots, \sigma_{N}^{(N)}\right)\right\} .
\end{aligned}
$$

Proof. This is merely a $2 N$-dimensional version of the Helly-Bray Theorem, and the proof follows that of the one-dimensional case. We omit the details.

Theorem 8. Corollary. If $f\left(\xi_{1}, \cdots, \xi_{2 N}\right)$ is bounded and continuous on the set $\left\{\left(\xi_{1}, \cdots, \xi_{2 N}\right): \xi_{j} \geqq \xi_{j+N}, 1 \leqq j \leqq N\right\}$ in $2 N$-dimensional Euclidean space, the conclusion of Theorem 8 is still true.

Proof. It suffices to observe that in the expectations of Theorem 8 , the arguments of $f$ are necessarily confined by $\xi_{j} \geqq \xi_{j+N}, 1 \leqq j \leqq N$. Since the set in question is closed, $f$ can be extended to a continuous bounded function on all of $2 N$-dimensional Euclidean space, agreeing with $f$ whenever $\xi_{j} \geqq \xi_{N+j}$, $1 \leqq j \leqq N$, and the corollary follows, applying Theorem 8 to the extended function.

The results of Theorem 8 and its corollary can be further extended to imply the convergence in distribution of $F\left[x_{n}(\cdot, \omega)\right]$ to $F[x(\cdot, \omega)]$ for a large class of functionals $F[$ ].

Theorem 9. Let $F[$ ] be a functional defined, bounded, and uniformly continuous in the uniform topology on the space $D$ of real functions defined on $[0,1]$, having the property that if $\left\{f_{n}, n \geqq 1\right\}$ is a sequence of functions of $D$ converging boundedly to $f$ in $D$, save for an at most countable subset of $[0,1]$, then

$$
\lim _{n \rightarrow \infty} F\left[f_{n}\right]=F[f]
$$

Then

$$
\lim _{n \rightarrow \infty} E\left\{F\left[x_{n}(\cdot, \omega)\right]\right\}=E\{F[x(\cdot, \omega)]\} .
$$

Proof. For each positive integer $N$, let

$$
\left.\begin{array}{l}
g_{N}^{*}(t)=\sup _{(g-1) / N<t \leqq j / N} g(t) ; \\
g_{N}^{* *}(t)=\inf _{(g-1) / N<t \leqq j / N} g(t)
\end{array}\right\} \quad \frac{j-1}{N}<t \leqq \frac{j}{N}, 1 \leqq j \leqq N .
$$

Let 


$$
M_{N, o}=\left\{h \in D ; g_{N}^{* *}(t) \leqq h(t) \leqq g_{N}^{*}(t)\right\}
$$

and let

$$
F_{N}^{*}[g]=\sup _{h \in m_{N, o}} F[h], \quad F_{N}^{* *}[g]=\inf _{h \in m_{N, o}} F[h] .
$$

We first observe that

$$
p\left\{\lim _{n \rightarrow \infty} F_{N}^{*}[x(\cdot, \omega)]=F[x(\cdot, \omega)]\right\}=1
$$

and

$$
p\left\{\lim _{n \rightarrow \infty} F_{N}^{* *}[x(\cdot, \omega)]=F[x(\cdot, \omega)]\right\}=1
$$

since the sample functions of $\{x(t, \omega), t \in[0,1]\}$ are almost all bounded with at most countably many jump discontinuities; application is made here of the special convergence property assumed for $F\left[\right.$ ]. Since $F_{N}^{* *}, F_{N}^{*}$, and $F$ can be bounded uniformly on $D$, we then have, by bounded convergence,

$$
\lim _{n \rightarrow \infty} E\left\{F_{N}^{* *}[x(\cdot, \omega)]\right\}=E\{F[x(\cdot, \omega)]\}
$$

and

$$
\lim _{n \rightarrow \infty} E\left\{F_{N}^{*}[x(\cdot, \omega)]\right\}=E\{F[x(\cdot, \omega)]\}
$$

Let now

$$
\begin{aligned}
f\left(\xi_{1}, \cdots, \xi_{2 N}\right) & =\sup _{\xi_{N+j} \leqq h(t) \leqq \xi_{j}} F[h], \quad \frac{j-1}{N}<t \leqq \frac{j}{N}, 1 \leqq j \leqq N \\
& =0 \text { if the conditions on the supremum are vacuous. }
\end{aligned}
$$

$f$ is clearly bounded on the set $\left\{\left(\xi_{1}, \cdots, \xi_{2 N}\right) ; \xi_{j} \geqq \xi_{N+j}, 1 \leqq j \leqq N\right\}$ and zero elsewhere. To apply Theorem 8 (Corollary) it is necessary to show that $f$ is continuous.

Given $\epsilon>0$ there is a $\delta>0$ such that for any $h_{1}, h_{2} \in D$, for which $\sup _{0 \leqq t \leqq 1}\left|h_{1}(t)-h_{2}(t)\right|<\delta$ we have

$$
\left|F\left[h_{1}\right]-F\left[h_{2}\right]\right|<\epsilon / 2 .
$$

Let $\left\{\xi_{1}, \cdots, \xi_{2 N}\right\}$ be any point for which $\xi_{j} \geqq \xi_{N+j}, 1 \leqq j \leqq N$. Let $g(t)$ be any function of $D$ for which

$$
\begin{aligned}
\xi_{j} & =\sup _{(j-1) / N<t \leqq J / N} g(t), \quad(1 \leqq j \leqq N) ; \\
\xi_{N+j} & =\inf _{(g-1) / N<t \leqq J / N} g(t) .
\end{aligned}
$$


Let $\left\{\hat{\xi}_{1}, \cdots, \hat{\xi}_{2 N}\right\}$ be any point such that $\hat{\xi}_{j} \geqq \hat{\xi}_{j+N}$ for $1 \leqq j \leqq N$, and such that

$$
\max _{1 \leqq j \leqq 2 N}\left|\xi_{j}-\widehat{\xi}_{j}\right|<\delta .
$$

Let $\hat{g}(t)$ be any element of $D$ such that

$$
\begin{aligned}
\widehat{\xi}_{j} & =\sup _{(j-1) / N<t<j / N} \hat{g}(t), \\
\hat{\xi}_{j+N} & =\inf _{(j-1) / N<t \leqq J / N} \hat{g}(t),
\end{aligned}
$$

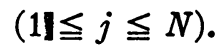

Choose $h_{1} \in M_{N, o}$ such that

$$
0 \leqq F_{N}^{*}[g]-F\left[h_{1}\right]<\frac{\epsilon}{2}
$$

and let

$$
\begin{aligned}
h_{2}(t) & =h_{1}(t), \hat{g}_{N}^{* *}(t) \leqq h_{1}(t) \leqq \hat{g}_{N}^{*}(t) \\
& =\widehat{g}_{N}^{* *}(t), h_{1}(t)<\hat{g}_{N}^{* *}(t) \\
& =\hat{g}_{N}^{*}(t), h_{1}(t)>\hat{g}_{N}^{*}(t) .
\end{aligned}
$$

Then $h_{2} \in M_{N, \hat{g}}$, so that $F\left[h_{2}\right] \leqq F_{N}^{*}[\hat{g}]$. Moreover,

$$
\sup _{0 \leqq t \leqq 1}\left|h_{1}(t)-h_{2}(t)\right|<\delta
$$

so that $\left|F\left[h_{1}\right]-F\left[h_{2}\right]\right|<\epsilon / 2$ and hence

$$
F_{N}^{*}[g]-F_{N}^{*}[\hat{g}]=\left(F_{N}^{*}[g]-F\left[h_{1}\right]\right)+\left(F\left[h_{1}\right]-F_{N}^{*}[\hat{g}]\right)<\frac{\epsilon}{2}+\frac{\epsilon}{2}=\epsilon .
$$

From the symmetry of the above argument in $h_{1}$ and $h_{2}$ it appears that $\left|F_{N}^{*}[g]-F_{N}^{*}[\hat{g}]\right|<\epsilon$. Since

$$
\begin{aligned}
& f\left(\xi_{1}, \cdots, \xi_{2 N}\right)=F_{N}^{*}[g], \\
& f\left(\hat{\xi}_{1}, \cdots, \hat{\xi}_{2 N}\right)=F_{N}^{*}[\hat{g}]
\end{aligned}
$$

this demonstrates the continuity of $f$.

Applying Theorem 8 (Corollary) to $f$ as constructed above, we conclude

$$
\lim _{n \rightarrow \infty} E\left\{F_{N}^{*}\left[x_{n}(\cdot, \omega)\right]\right\}=E\left\{F_{N}^{*}[x(\cdot, \omega)]\right\} .
$$

Similarly, one may show

$$
\lim _{n \rightarrow \infty} E\left\{F_{N}^{* *}\left[x_{n}(\cdot, \omega)\right]\right\}=E\left\{F_{N}^{* *}[x(\cdot, \omega)]\right\} .
$$

We therefore have 


$$
\begin{aligned}
E\{F x(\cdot, \omega)]\} & =\lim _{n \rightarrow \infty} E\left\{F_{N}^{* *}[x(\cdot, \omega)]\right\}=\lim _{n \rightarrow \infty} \lim _{n \rightarrow \infty} E\left\{F_{N}^{* *}\left[x_{n}(\cdot, \omega)\right]\right\} \\
& \leqq \liminf _{n \rightarrow \infty} E\left\{F\left[x_{n}(\cdot, \omega)\right]\right\} \leqq \lim _{n \rightarrow \infty} \sup E\left\{F\left[x_{n}(\cdot, \omega)\right]\right\} \\
& \leqq \lim _{N \rightarrow \infty} \lim _{n \rightarrow \infty} E\left\{F_{N}\left\lfloor x_{n}\left(\cdot^{*}, \omega\right)\right]\right\} \\
& =\lim _{N \rightarrow \infty} E\left\{F_{N}^{*}[x(\cdot, \omega)]\right\}=E\{F[x(\cdot, \omega)]\}
\end{aligned}
$$

which completes the proof.

Theorem 9. Corollary. If $F[$ ] is a functional defined on $D$, uniformly continuous in the uniform topology on $D$, and such that if $\left\{f_{n}, n \geqq 1\right\} \subset D$ is a sequence converging to $f \in D$ boundedly except for a countable subset of $[0,1]$, then $\lim _{n \rightarrow \infty} F\left[f_{n}\right]=F[f]$, then

$$
\lim _{n \rightarrow \infty} p\left\{F\left[x_{n}(\cdot, \omega)\right] \leqq \lambda\right\}=p\{F[x(\cdot, \omega)] \leqq \lambda\}
$$

at continuity points of the function on the right.

Proof. Applying Theorem 9 to $\cos \mu F\left[x_{n}(\cdot, \omega)\right]$ and $\sin \mu F\left[x_{n}(\cdot, \omega)\right]$, we conclude

$$
\lim _{n \rightarrow \infty} E\left\{\exp i \mu F\left[x_{n}(\cdot, \omega)\right]\right\}=E\{\exp i \mu F[x(\cdot, \omega)]\}
$$

from which the desired result follows.

The Corollary to Theorem 9 is an extension of a result due to Donsíer [3], wherein the limiting process $\{x(t, \omega), t \in[0,1]\}$ is the Wiener process In general if the limiting process is Gaussian, one obtains the following:

TheOREM 10. Let $\{x(t, \omega), t \in[0,1]\}$ be Gaussian, and let $F$ be a continuous functional in the uniform topology on $D$. Then at continuity points $\lambda$ of $p\{F[x(\cdot, \omega)] \leqq \lambda\}$ we have

$$
\lim _{n \rightarrow \infty} p\left\{F\left[x_{n}(\cdot, \omega)\right] \leqq \lambda\right\}=p\{F[x(\cdot, \omega)] \leqq \lambda\} .
$$

Proof. Since the sample functions of a separable Gaussian process with independent increments are almost all continuous, it follows that for a functional $F$ satisfying the conditions of this theorem one has

$$
\begin{gathered}
p\left\{\lim _{N \rightarrow \infty} F_{N}^{*}[x(\cdot, \omega)]=F[x(\cdot, \omega)]\right\}=1, \\
p\left\{\lim _{N \rightarrow \infty} F_{N}^{* *}[x(\cdot, \omega)]=[x(\cdot, \omega)]\right\}=1
\end{gathered}
$$

in the proof of Theorem 9. The rest of the proof of Theorem 9 demonstrates the validity of the conclusion of Theorem 9 for bounded uniformly continuous 
$F[$ ]; since any bounded continuous functional can be approximated in the mean on a Gaussian process by bounded uniformly continuous functionals, the result of Theorem 9 extends to bounded continuous functionals. The method of the Corollary to Theorem 9 establishes the desired conclusion. For the details of this proof see [3].

It is to be observed that since any separable Gaussian process with independent increments, no fixed points of discontinuity, and parameter set $[a, b]$ can, if $p\{x(a, \omega)=0\}=1$, be transformed by a change of scale into the separable Wiener process, the result of Theorem 10 has specious generality over that of [3].

5. Remarks. From Theorem 5, it appears that the sequence $(A)$ under condition (C) satisfies the conditions of the theorems in $\$ 4$ on the processes $\left\{x_{n}(t, \omega), t \in[0,1]\right\}$ and $\{x(t, \omega), t \in[0,1]\}$, and the results of Theorems 7-10 therefore apply to this particular sequence. Moreover, if $\{x(t, \omega), t \in[0,1]\}$ is a stochastic process with independent increments and no fixed points of discontinuity, it appears that if

$$
x_{n k}=x\left(\frac{k}{n}, \omega\right)-x\left(\frac{k-1}{n}, \omega\right), \quad 1 \leqq k \leqq n, n \geqq 1
$$

then $\left\{x_{n k}, 1 \leqq k \leqq n\left(=k_{n}\right), n \geqq 1\right\}$ generates a sequence of the type $(A)$ converging uniformly in distribution to $\{x(t, \omega), t \in[0,1]\}$, and the force of Theorems $7-10$ can be used as a device in the calculation of $p\{F[x(\cdot, \omega)] \leqq \lambda\}$, establishing the method most commonly used for this purpose in general.

\section{REFERENCES}

1. T. W. Anderson and D. A. Darling, Asymptotic theory of certain goodness of fit criteria based on stochastic processes, Ann. Math. Statist. vol. 23 (1952) pp. 193-212.

2. M. D. Donsker, An invariance principle for certain probability limit theorems, Memoirs of the American Mathematical Society, no. 6, 1951, pp. 1-12.

3. - Justification and extension of Doob's heuristic approach to the KolmogorovSmirnov theorems, Ann. Math. Statist. vol. 23 (1952) pp. 277-281.

4. J. Doob, Heuristic approach to the Kolmogorov-Smirnov theorems, Ann. Math. Statist. vol. 20 (1949) pp. 393-403.

5. - Stochastic processes, Wiley, 1953.

6. B. V. Gnedenko and A. N. Kolmogorov, Lirriti disiriviutiviis füi juiiiij of iiidéperidert random variables, Addison-Wesley, 1954.

7. M. Kac and P. Erdös, On certain limit theorems of the theory of probability, Bull. Amer. Math. Soc. vol. 52 (1946) pp. 292-302.

8. - On the number of positive sums of independent random variables, Bull. Amer. Math. Soc. vol. 53 (1947) pp. 1011-1020.

9. M. Kac, On some connections between probability theory and differential and integral equations, Proceedings of the Second Berkeley Symposium on Mathematical Statistics and Probability, University of California Press, Berkeley and Los Angeles, 1950, pp. 189-216.

UNIVERSITY OF MINNESOTA, MinNEAPOLIS, MinN. 